\title{
Pressure Sensor for Remote Readout in Aqueous Environment
}

\author{
Torben Karrock and Martina Gerken \\ Institute of Electrical Engineering and Information Technology, \\ Christian-Albrechts-Universität zu Kiel, Kaiserstr. 2, 24143 Kiel, Germany, \\ tok@tf.uni-kiel.de,mge@tf.uni-kiel.de
}

\begin{abstract}
:
Implantable intraocular pressure sensors are of high interest for early diagnosis of glaucoma. We propose a miniaturized, remote-readout, pressure-sensitive element that can be integrated into an artificial intraocular lens. A reference volume is sealed with a flexible photonic-crystal membrane. The optical spectrum reflected from this nanostructured waveguide depends on the incidence angle. A deformation of the membrane is calculated from an RGB image. Signal drift is a challenge with this type of membrane sensor. Here, we investigate the stability of the sensor for operation in air and in water. We identify gas leakage as the main reason of the signal drift, which is significantly reduced in an aqueous environment.
\end{abstract}

Key words: pressure sensors, glaucoma, flexible photonic crystal, resonant waveguide grating, membrane, stability

\section{Introduction}

Glaucoma is one of the leading causes of blindness. A high intraocular pressure is the main symptom of glaucoma. Currently, tonometry is the method of choice for intraocular pressure measurements. There are different kinds of measurement methods but all of them need to be performed by trained medical staff. Especially during the medication adjustment for the patients, measurements have to be performed regularly. This costs time and is not very comfortable for the patients.

Thus, the interest in an implantable intraocular pressure sensors, which can be read out by the patient himself, is high. Different techniques have been suggested. The two main groups can be divided into electronic sensors that are powered from the outside [1,2] and powerless sensors that directly transfer the pressure via a deformation into a parameter that can be read out optically [3-6]. Our sensor belongs to the second group. We propose to enhance artificial intraocular lenses by including a pressuresensitive element. This could be introduced at little extra effort during a standard intraocular lens replacement surgery, which is one of the most common eye surgeries.

Fig. 1 depicts our system concept. The pressure-sensitive element consists of a reference volume sealed with a flexible photonic-crystal membrane [6]. The intraocular pressure deforms the membrane and changes the viewing angle for different regions of the membrane with respect to the optical axis of the imaging readout system. The nanostructured photonic-crystal membrane exhibits angledependent resonances in the spectrum that cause a specific color impression in the image. The color coding is enhanced, when the background is suppressed with crossed polarization filters [7]. When the viewing angle changes with membrane deformation, the resonance position does so, too. Therefore, a specific color pattern is observed depending on the pressure value. Here, we investigate the drift stability of the sensor prototype in air and in water.

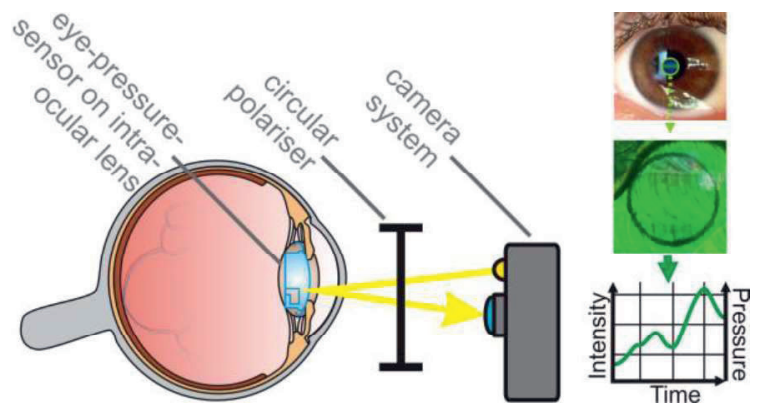

Fig. 1: Concept of artificial intraocular lens enhanced with pressure-sensitive element for noncontact, remote-readout of the intraocular pressure. Deformation of a nanostructured photonic crystal membrane is detected with a camera system. 


\section{Fabrication and characterization methods}

The sensor prototype is fabricated in polydimethylsiloxane (PDMS, Sylgard 184 from Sigma Aldrich) employing two molds and subsequent joining of the two parts. In one mold a thin membrane with an imprinted linear nanostructure is fabricated by pressing a previously produced nanostructured stamp into the uncured PDMS in the mold. The nanostructured stamp is fabricated with a lithographic process from an electron-beam written glass master with $400 \mathrm{~nm}$ grating period (from AMO GmbH, Aachen, Germany).

A high index waveguide is than applied by spin coating a randomly arranged $\mathrm{TiO}_{2}$-nanoparticle layer (titanium(IV) oxide, mixture of rutile and anatase, 33-37 wt $\%$ in $\mathrm{H}_{2} \mathrm{O}$ from SigmaAldrich, St. Louis, Missouri, USA; diameter $<150 \mathrm{~nm}$; $\sim 21 \mathrm{~nm}$ primary particle size of starting nanopowder) on top of the cured nanostructured PDMS membrane [6]. With this process a flexible photonic crystal with a resonance peak in the green region of the visible spectrum is fabricated. Fig. 2 shows a schematic of the sensor.

Note that the prototype is up-scaled by a factor of approximately 4 and that the lens function is not included. Nevertheless, this prototype is suitable for testing the performance. Fig. 3 depicts the characterization setup. The pressure chamber is filled with air or water.

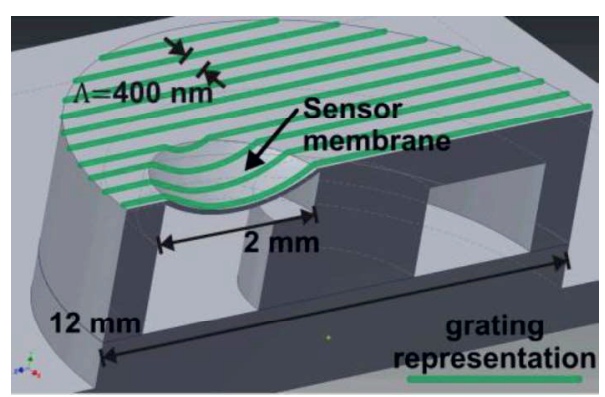

Fig. 2: Schematic of sensor prototype.

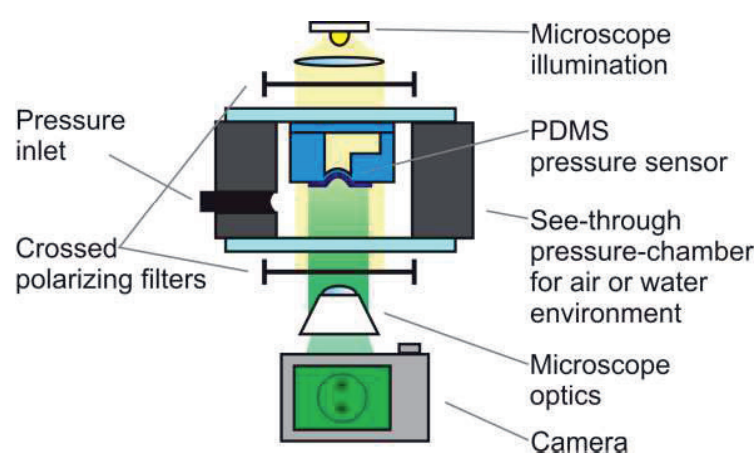

Fig. 3: Characterization setup. The pressure chamber may be filled with air or water.

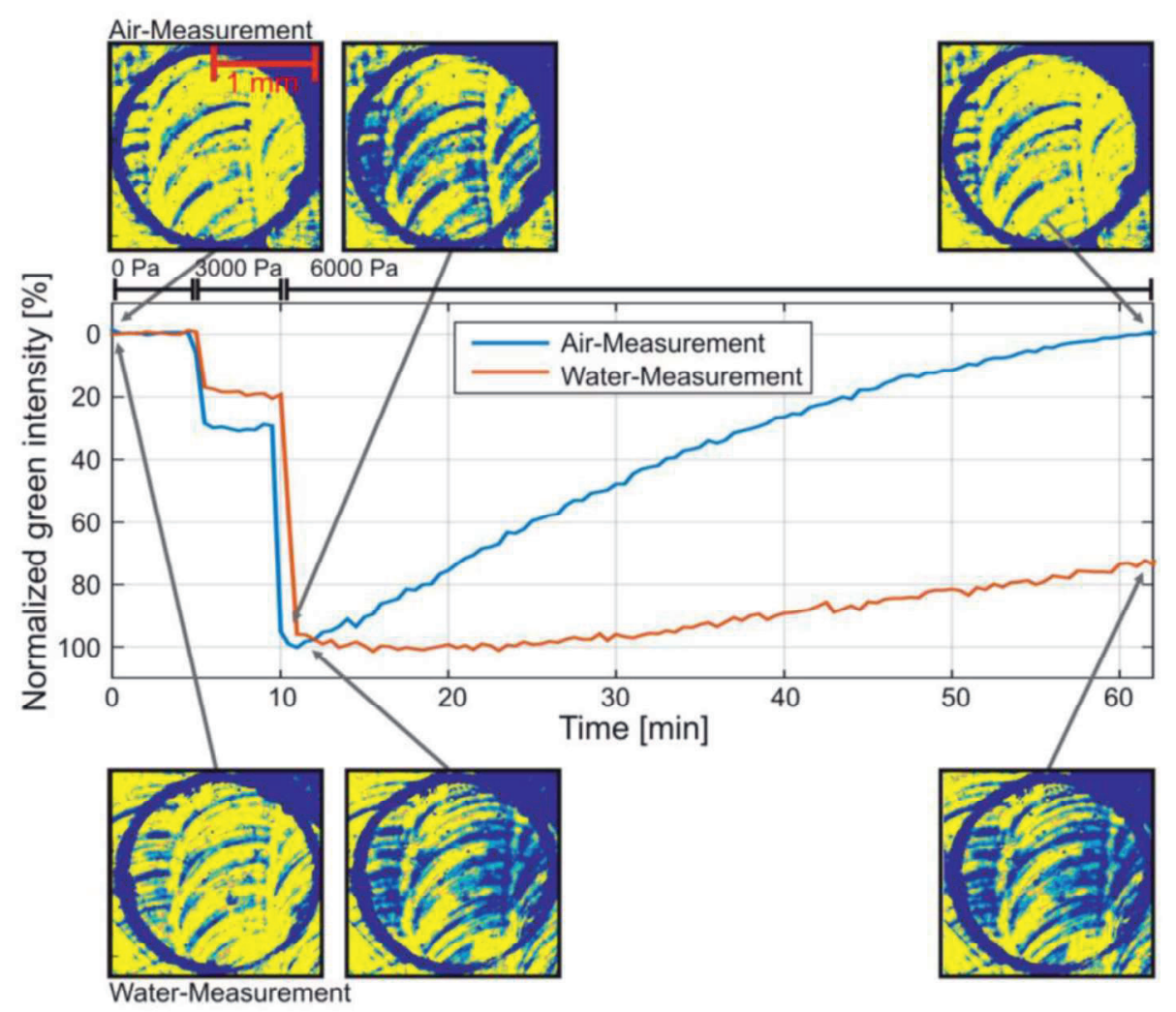

Fig. 4: Sensor signal for three different pressure values (0 Pa, $2000 \mathrm{~Pa}, 6000 \mathrm{~Pa}$ ) in air (blue graph) and in water (red graph). The six small inlays show the intensity of the green color channel when imaging the photonic crystal membrane. The colormap is adjusted to better show the intensity reduction through the deformation and does not represent exact numbers. 


\section{Results}

Figure 4 shows the measurement results for operation of the sensor in air and in water. The pressure is changed from $0 \mathrm{~Pa}$ over $3000 \mathrm{~Pa}$ to $6000 \mathrm{~Pa}$. The intensity change in the green color-channel of the camera is monitored. The six inlays in Figure 4 show the intensity of the green color channel while observing the photonic crystal membrane. The colormap was adjusted to better show the intensity reduction through the deformation and does not represent exact numbers. Please note that the circular features originate from the milling process of the mold.

The color change upon a pressure change is clearly visible. Also visible is a large drift of the signal for one-hour operation with air in the pressure chamber. The drift is significantly reduced when operating the sensor in an aqueous environment. We attribute the drift to gas leakage. It is known that PDMS shows gas permeability $[8,9]$. While the signal drift within one hour is $\sim 100 \%$ for operation in air, it still amounts to $\sim 25 \%$ in water.

\section{Conclusions}

In conclusion, it is shown that the concept of a pressure-sensitive element integrated with an artificial intraocular lens is viable. In the material choice, low gas leakage is highly important besides factors such as biocompatibility, transparency, and flexibility. Therefore, the next step is to investigate other materials for realizing the intraocular pressure sensor. Alternatively, the membrane may be sealed against gas leakage to allow for stable operation.

\section{References}

[1] W. Mokwa, Meas. Sci. Technol. 18(5), R47-R57 (2007); doi: 10.1088/0957-0233/18/5/R01

[2] N. Xue, J.-B. Lee, S. Foland, S. P. Chang, IEEE Sensors Proc. 1930, 1748-1751 (2011); doi: 10.1109/ICSENS.2011.6127005

[3] P.-J. Chen, D. C. Rodger, R. Agrawal, S. Saati, E. Meng, R. Varma, M. S. Humayun, Y.-C. Tai, J. Micromech. Microeng. 17(10), 1931-1938 (2007); doi: 0.1088/0960-1317/17/10/002

[4] I. E. Araci, B. Su, S. R. Quake, Y. Mandel, Nat. Med. 20(9), 1074-1078 (2014), doi: $10.1038 / \mathrm{nm} .3621$

[5] Y. Nazirizadeh, T. Karrock, M. Gerken, Opt. Lett. 37(15), 3081-3083 (2012); doi: 10.1364/OL.37.003081

[6] T. Karrock, M. Gerken, Biomedical Optics Express 6(12), 4901-4911 (2015); doi: 10.1364/BOE.6.004901

[7] Y. Nazirizadeh, J. G. Müller, U. Geyer, D. Schelle, E. -. Kley, A. Tünnermann, U. Lemmer, M. Gerken, Opt. Express 16(10), 7153-7160. (2008); doi: 10.1364/OE.16.007153

[8] J. Goldowsky,H. F. Knapp, RSC Advances 3, 17968-17976 (2013); doi: 10.1039/c3ra42977f

[9] T. C. Merkel, V. I. Bondar, K. Nagai, B. D. Freeman, I. Pinnau, J. Polym. Sci. B Polym. Phys. 38: 415-434 (2000); doi: $10.1002 /(\mathrm{SICl}) 1099-$ 0488(20000201)38:3<415::AIDPOLB8>3.0.CO;2-Z 